\title{
Inverse Compton X-rays from relativistic flare electrons and positrons
}

\author{
A. L. MacKinnon and P. C. V. Mallik \\ Department of Physics and Astronomy, University of Glasgow, Glasgow G12 8QQ, UK \\ e-mail: alec@astro.gla.ac.uk
}

Received 26 August 2009 / Accepted 6 November 2009

\begin{abstract}
Context. In solar flares, inverse Compton scattering (ICS) of photospheric photons might give rise to detectable hard X-ray photon fluxes from the corona where ambient densities are too low for significant bremsstrahlung or recombination. $\gamma$-ray lines and continuum in some large flares imply the presence of the necessary $\sim 100 \mathrm{MeV}$ electrons and positrons, the latter as by-products of GeV energy ions. Recent observations of coronal hard X-ray sources in particular prompt us to reconsider here the possible contribution of ICS.

Aims. We aim to evaluate the ICS X-ray fluxes to be expected from prescribed populations of relativistic electrons and positrons in the solar corona. The ultimate aim is to determine if ICS coronal X-ray sources might offer a new diagnostic window on relativistic electrons and ions in flares.

Methods. We use the complete formalism of ICS to calculate X-ray fluxes from possible populations of flare primary electrons and secondary positrons, paying attention to the incident photon angular distribution near the solar surface and thus improving on the assumption of isotropy made in previous solar discussions.

Results. Both primary electrons and secondary positrons produce very hard ICS X-ray spectra. The anisotropic primary radiation field results in pronounced centre-to-limb variation in predicted fluxes and spectra, with the most intense spectra, extending to the highest photon energies, expected from limb flares. Acceptable numbers of electrons or positrons could account for RHESSI coronal $\mathrm{X} / \gamma$-ray sources.

Conclusions. Some coronal X-ray sources at least might be interpreted in terms of ICS by relativistic electrons or positrons, particularly when sources appear at such low ambient densities that bremsstrahlung appears implausible.
\end{abstract}

Key words. acceleration of particles - radiation mechanisms: general - Sun: corona - Sun: photosphere - Sun: flares Sun: X-rays, gamma rays

\section{Introduction}

Korchak $(1967,1971)$ considered three possible radiation mechanisms via which solar flare energetic electrons might produce hard X-rays (HXRs): synchrotron, bremsstrahlung and inverse Compton scattering (ICS). He established that fluxes from electron-ion bremsstrahlung would dominate those from the other two mechanisms under normal solar atmosphere conditions and thus laid one of the foundations of the interpretation of flare X-rays. Left open, however, was the possibility that ICS HXR fluxes from low-density regions might exceed those from bremsstrahlung (or, indeed, recombination - Brown \& Mallik 2008, 2009). Recent years have seen increasingly detailed observations of coronal HXR sources (Hudson et al. 2001; Krucker et al. 2008a,b; Tomczak 2009), sometimes from surprisingly tenuous regions. Reconsideration of the possible role of ICS in HXR production thus seems timely (Krucker et al. 2008a).

The basics of ICS are well understood (e.g. Blumenthal \& Gould 1970; Rybicki \& Lightman 1986). Suppose that electrons of a (total) energy $\gamma m_{\mathrm{e}} c^{2}$ scatter photons of an initial energy $\epsilon_{\mathrm{i}}$. Optical photons of photospheric origin, for instance, would have $\epsilon_{\mathrm{i}}$ typically of the order of $2 \mathrm{eV}$. The maximum possible scattered photon energy results from a head-on collision of electron and photon and has a value of $\epsilon_{\max } \simeq 4 \gamma^{2} \epsilon_{\mathrm{i}}$ (e.g. Blumenthal \& Gould 1970).
To produce HXR photons via ICS of optical photons thus needs electrons in the $10 \mathrm{~s}$ to $100 \mathrm{~s}$ of $\mathrm{MeV}$ energy range.

There is good evidence that electrons attain such energies in flares. Gamma-ray continuum radiation in this energy range has been observed from some large flares (e.g. Forrest \& Chupp 1983; Kanbach et al. 1993; Talon et al. 1993). Electron-ion bremsstrahlung from primary accelerated electrons may contribute to this feature, as may bremsstrahlung from secondary electrons and positrons in the $100 \mathrm{MeV}$ energy range, produced in reactions of accelerated ions in the energy range $>0.3 \mathrm{GeV}$ (e.g. Murphy et al. 1987). In the latter case, positrons are dominant in number since they result from collisions between positively charged particles. The continuum radiation in this case is unavoidably accompanied by the flat spectral feature around $70 \mathrm{MeV}$ produced by $\pi^{0}$ decay. High-energy radiation can occur both with and without this feature at different times during a single event (e.g. Vilmer et al. 2003), indicating that both primary accelerated electrons and secondary positrons may be present in the $100 \mathrm{MeV}$ energy range, as needed for ICS HXR production. Akimov et al. (1994) give evidence that the flare of 26 March 1991 accelerated electrons to energies of $300 \mathrm{MeV}$. The energy distributions of electrons and positrons will be very different, however, and we consider them separately.

In the presence of the solar magnetic field, these high energy electrons would also produce synchrotron emission, but at radio 
and sub-mm wavelengths (Silva et al. 2007). To produce X-rays by synchrotron emission would require electrons of unrealistically high energy, for which there is no evidence.

The ICS estimates of Korchak $(1967,1971)$ and Krucker et al. (2008a) employ standard results based on assuming isotropic electron and photon distributions. Electron distributions in the corona may well be isotropic because of pitch-angle scattering by MHD turbulence (e.g. Miller \& Ramaty 1989), but the photon distribution will be isotropic only in the outward hemisphere. As already mentioned, the most energetic photons result from head-on collisions of photon and electron, which result in the up-scattered photon travelling along the direction of the incident electron (Jones 1968). These most favourable collisions clearly cannot occur, even with an assumed isotropic coronal electron distribution, so a more involved calculation is essential to evaluate likely ICS fluxes, spectra etc.

ICS is certainly important in other areas of astrophysics: for instance, cosmic microwave background photons are scattered by hot gas in clusters of galaxies (Sunyaev-Zeldovich effect Sunyaev \& Zeldovich 1970) and solar visible photons are scattered by cosmic ray electrons (Orlando \& Strong 2008).

The formalism for calculating ICS radiation with arbitrary photon angular distributions has been given most recently by Moskalenko \& Strong (2000). Here we adapt their work to the source geometry near the solar surface. We use typically observed power-law distributions of electrons and protons (which produce secondary positrons) to illustrate our study. We elucidate the consequences for the observability of this ICS flux and note the difference between the spectra produced by electrons and by secondary positrons, as well as the disc-centre to limb variation. Our findings reveal that although the ICS intensities are likely to be low, the spectrum is hard and unmistakable. If detected by modern instruments, this would be a new window on extremes of electron and ion acceleration at the Sun, and in the case of ions complementing information available from $\gamma$-ray lines and free neutrons detected in space.

In this paper, we use the units $\hbar=c=m_{\mathrm{e}}=1$.

\section{Source geometry; calculation of IC flux}

In this section we calculate the ICS HXR fluxes from relativistic electron and positron populations in the corona, following Moskalenko \& Strong (2000).

The rate of photon-particle interactions is given in full generality by (Weaver 1976):

$R=n_{\mathrm{e}} n_{\gamma} \int \mathrm{d} \boldsymbol{p}_{\gamma} \int \mathrm{d} \boldsymbol{p}_{\mathrm{e}} f_{\mathrm{e}}\left(\boldsymbol{p}_{\mathrm{e}}\right) f_{\gamma}\left(\boldsymbol{p}_{\gamma}\right) \frac{p_{\gamma}^{\prime}}{\gamma p_{\gamma}} \sigma\left(p_{\gamma}^{\prime}\right)$,

where $n_{\mathrm{e}}, n_{\gamma}$ are the electron and photon number densities; $\boldsymbol{p}_{\mathrm{e}}$, $\boldsymbol{p}_{\gamma}$ are the momenta; $f_{\mathrm{e}}\left(\boldsymbol{p}_{\mathrm{e}}\right), f_{\gamma}\left(\boldsymbol{p}_{\gamma}\right)$ are the respective distribution functions in the laboratory system (LS), normalised to unity; $\gamma$ is the electron Lorentz factor; $\sigma$ the cross-section and the primes signify the electron rest system (ERS) variables. For relativistic electrons, the incoming photons are seen as a narrow beam $\sim 1 / \gamma$ wide in the ERS. We follow Moskalenko \& Strong (2000) in using the Jones (1968) approximation that the incident photons are seen as a unidirectional beam in the ERS. This significantly simplifies the calculation of the ICS fluxes while introducing negligible error (Jones 1968).

To calculate fluxes from Eq. (1), we need to specify the electron and photon momentum distributions and the cross-section. Since we deal with highly relativistic particles and situations

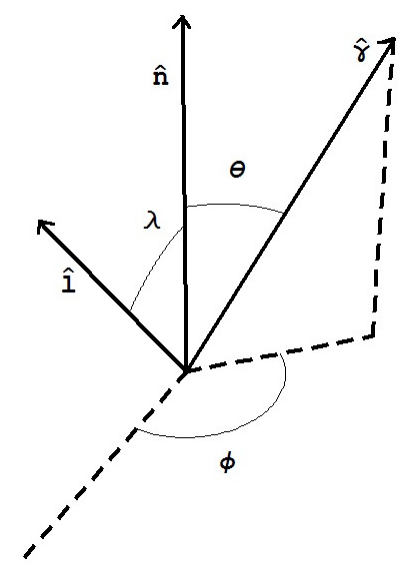

Fig. 1. Schematic diagram showing the geometry used to describe the radiation field at the solar surface with the relevant angles and vectors. $\phi$ lies in the solar surface plane.

where the photon may carry away a large fraction of the electron energy, we must use the Klein-Nishina cross-section (e.g. Jauch \& Rohrlich 1976):

$$
\begin{aligned}
\frac{\mathrm{d} \sigma}{\mathrm{d} \epsilon_{2}^{\prime} \mathrm{d} \cos \eta^{\prime}}= & \pi r_{\mathrm{e}}^{2}\left(\frac{\epsilon_{2}^{\prime}}{\epsilon_{1}^{\prime}}\right)^{2}\left(\frac{\epsilon_{2}^{\prime}}{\epsilon_{1}^{\prime}}+\frac{\epsilon_{1}^{\prime}}{\epsilon_{2}^{\prime}}-\sin ^{2} \eta^{\prime}\right) \\
& \times \delta\left[\epsilon_{2}^{\prime}-\frac{\epsilon_{1}^{\prime}}{1+\epsilon_{1}^{\prime}\left(1-\cos \eta^{\prime}\right)}\right],
\end{aligned}
$$

where $r_{\mathrm{e}}$ is the classical electron radius, $\epsilon_{1}^{\prime}$ and $\epsilon_{2}^{\prime}$ are the ERS energies of the incident and up-scattered photons, $\eta^{\prime}$ is the scattering angle in the ERS and $\delta(x)$ denotes the Dirac delta function.

Appropriately to the general galactic cosmic ray population, Moskalenko \& Strong (2000) assume isotropic electrons; this assumption will also be appropriate in the corona as a result of MHD scattering (Miller \& Ramaty 1989; Mandzhavidze \& Ramaty 1992). With these assumptions, the up-scattered photon distribution over the LS energy, $\epsilon_{2}$, as obtained from Eq. (1) is (Moskalenko \& Strong 2000)

$$
\begin{aligned}
\frac{\mathrm{d} R}{\mathrm{~d} \epsilon_{2}}= & \int \mathrm{d} \cos \eta^{\prime} \int \mathrm{d} \epsilon_{1} \mathrm{~d} \Omega_{\gamma} \int \mathrm{d} \gamma \mathrm{d} \Omega_{\mathrm{e}} \\
& \times f_{\mathrm{e}}\left(\gamma, \Omega_{\mathrm{e}}\right) f_{\gamma}\left(\epsilon_{1}, \Omega_{\gamma}\right) \epsilon_{1}^{2} \gamma^{2} \frac{\epsilon_{1}^{\prime}}{\gamma \epsilon_{1}} \frac{\epsilon_{2}^{\prime}}{\epsilon_{2}} \frac{\mathrm{d} \sigma}{\mathrm{d} \epsilon_{2}^{\prime} \mathrm{d} \cos \eta^{\prime}},
\end{aligned}
$$

where $\Omega_{\gamma}$ and $\Omega_{\mathrm{e}}$ refer to photon and electron directions respectively.

At this point we depart from Moskalenko \& Strong (2000), tailoring our calculation to the radiation field geometry above the solar surface (Fig. 1). We introduce two, spherical polar angular coordinates $\theta$ and $\phi$ to label photon direction. Let $\hat{n}$ be a unit vector pointing radially outward from the local solar surface, and $\hat{l}$ be a unit vector pointing along the line of sight to the observer. Then we have $\hat{l} \cdot \hat{n}=\sin \lambda$, where $\lambda$ is the heliocentric angle of the source location. Let $\hat{p}_{\gamma}$ be a unit vector in the direction of the photon. The polar angle $\theta$ measures the angle between $\hat{n}$ and $\hat{p}_{\gamma}$, i.e. $\hat{n} \cdot \hat{p}_{\gamma}=\cos \theta$. The photon azimuthal angle $\phi$ lies in the plane of the solar surface and is measured anticlockwise from the plane defined by $\hat{n}$ and $\hat{l}$.

The photon distribution is isotropic in the optically thick photosphere, but only includes outward-flowing photons immediately above. It will be close to isotropic, in the hemisphere 
$\theta<\pi / 2$, as long as we consider coronal locations below $\sim 2 R_{\odot}$. Thus the photon angular distribution takes the simple form

$f_{\gamma}\left(\epsilon_{1}, \theta, \phi\right)=\frac{1}{2 \pi} H\left(\frac{\pi}{2}-\theta\right) g_{\gamma}\left(\epsilon_{1}\right)$,

where $H$ is the Heaviside step function.

In the first instance we calculate the ICS flux from monoenergetic electrons with a single energy $\gamma$, averaging straightforwardly over more general energy distributions as needed. We also consider monoenergetic primary photon distributions, $g_{\gamma}(x)=\delta\left(x-\epsilon_{1}\right)$. Using Eqs. (2)-(4), we hence find the total up-scattered photon distribution per electron over the LS energy, $\epsilon_{2}$, to be:

$$
\begin{aligned}
\frac{\mathrm{d} R}{\mathrm{~d} \epsilon_{2}}= & \left(2-\frac{4 \epsilon_{2}}{\gamma}+\frac{3 \epsilon_{2}^{2}}{\gamma^{2}}-\frac{\epsilon_{2}^{3}}{\gamma^{3}}\right) \int_{0}^{2 \pi} \int_{\theta_{\min }}^{\theta_{\max }} \sin \theta \mathrm{d} \theta \mathrm{d} \phi \\
& -\frac{1}{\epsilon_{1} \gamma}\left(\frac{2 \epsilon_{2}^{2}}{\gamma^{2}}-\frac{2 \epsilon_{2}}{\gamma}\right) \int_{0}^{2 \pi} \int_{\theta_{\min }}^{\theta_{\max }} \frac{\mathrm{d} \cos \theta}{1+\cos \theta} \mathrm{d} \phi \\
& -\frac{\epsilon_{2}^{2}}{\epsilon_{1}^{2} \gamma^{4}} \int_{0}^{2 \pi} \int_{\theta_{\min }}^{\theta_{\max }} \frac{\mathrm{d} \cos \theta}{(1+\cos \theta)^{2}} \mathrm{~d} \phi .
\end{aligned}
$$

The lower limit of the $\theta$ integral is given by kinematics:

$\theta_{\min }=\arccos \left(1-\frac{\epsilon_{2}}{2 \epsilon_{1} \gamma\left(\gamma-\epsilon_{2}\right)}\right)$

and the upper limit by source geometry:

$\theta_{\max }=\arccos (\sin \lambda \cos \phi)$.

Performing the integral over polar angle we get

$$
\begin{aligned}
\frac{\mathrm{d} R}{\mathrm{~d} \epsilon_{2}}= & \frac{r_{\mathrm{e}}^{2}}{2 \epsilon_{1}\left(\gamma-\epsilon_{2}\right)^{2}} \int_{0}^{2 \pi}\left(\frac{\epsilon_{2}^{3}}{\gamma^{3}}-\frac{3 \epsilon_{2}^{2}}{\gamma^{2}}+\frac{4 \epsilon_{2}}{\gamma}-2\right) \\
& \times\left(\sin \lambda \cos \phi-\left(1-\frac{\epsilon_{2}}{2 \epsilon_{1} \gamma\left(\gamma-\epsilon_{2}\right)}\right)\right)+\frac{2 \epsilon_{2}}{\epsilon_{1} \gamma^{2}}\left(1-\frac{\epsilon_{2}}{\gamma}\right) \\
& \times\left(\ln (1+\sin \lambda \cos \phi)-\ln \left(2-\frac{\epsilon_{2}}{2 \epsilon_{1} \gamma\left(\gamma-\epsilon_{2}\right)}\right)\right) \\
& +\frac{\epsilon_{2}^{2}}{\epsilon_{1}^{2} \gamma^{4}}\left(\frac{1}{1+\sin \lambda \cos \phi}-\frac{1}{2-\epsilon_{2} /\left(2 \epsilon_{1} \gamma\left(\gamma-\epsilon_{2}\right)\right)}\right) \mathrm{d} \phi,
\end{aligned}
$$

which is the ICS flux of photons per unit energy per unit time per electron. The following kinematic results (Moskalenko \& Strong 2000) are also of importance:

$\epsilon_{2}^{\prime}=\epsilon_{2} /\left[\gamma\left(1-\cos \eta^{\prime}\right)\right], \epsilon_{2} \leq 2 \gamma \epsilon_{1}^{\prime} /\left(1+2 \epsilon_{1}^{\prime}\right), \epsilon_{1}^{\prime}=\epsilon_{1} \gamma(1+\cos \lambda)$. (9)

The maximum energy of the up-scattered photon is

$\epsilon_{2 \max }=4 \epsilon_{1} \gamma^{2} /\left(1+4 \epsilon_{1} \gamma\right)$.

Note that the second and third terms in Eq. (8) have to be evaluated numerically. This was done using MATLAB, and the results are portrayed in the following section.

\section{Results}

\subsection{ICS from fast electrons}

To calculate ICS spectra produced by relativistic electrons, we assumed power-law primary electron kinetic energy distributions extending into the $10 \mathrm{~s}$ of $\mathrm{MeV}$ range, $\sim(\gamma-1)^{-\delta}$. The incident photon population was assumed to have a monoenergetic

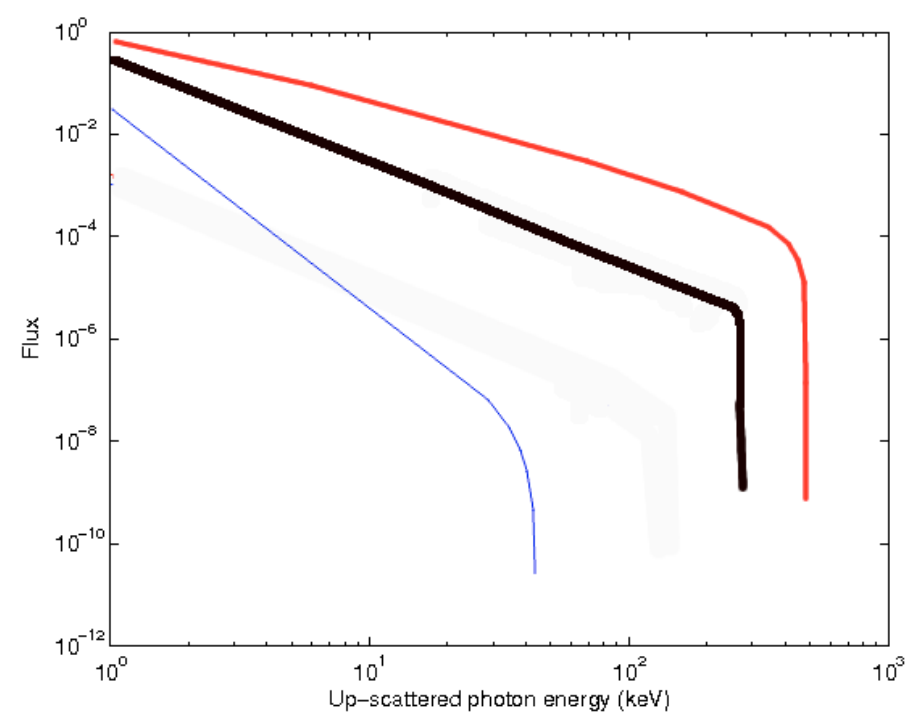

Fig. 2. Photon spectra at the Sun (photons per keV per second per source electron) from limb fast electrons with different power-law distributions: thin-black is for $\delta=5$, medium-red for $\delta=3$ and thick-blue for $\delta=2$ with an incident photon energy of $2 \mathrm{eV}$.

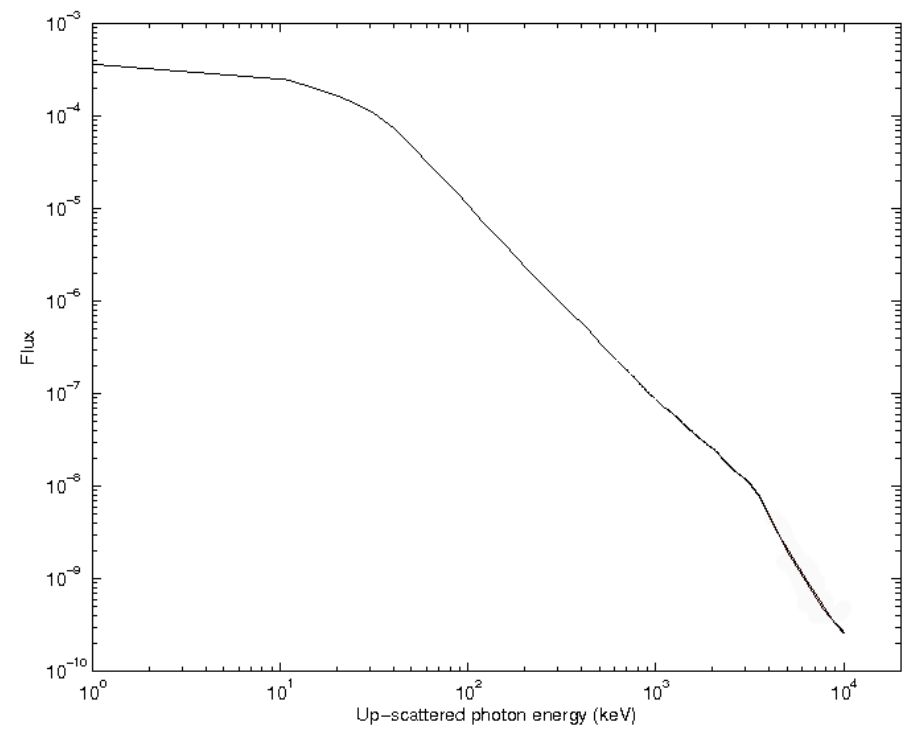

Fig. 3. Photon spectra at the Sun (photons per keV per second per source electron) from fast electrons with a power-law energy distribution $E^{-3}$ for an incident photon energy of $200 \mathrm{eV}$.

energy distribution at $\epsilon_{1}=2 \mathrm{eV}$ (or $200 \mathrm{eV}$ in a few, illustrative cases) so that the solar luminosity implies a photon density of $n_{\gamma}=10^{12} \mathrm{~cm}^{-3}$. We have checked that the spectra found using the full, black body photospheric spectrum are not significantly different from these shown here for the $2 \mathrm{eV}$ case.

In Fig. 2 we show the ICS spectra from electrons with an energy spectral index $\delta=3$, calculated by weighting the emissivity (8) by this distribution and integrating over electron energy. Fluxes are normalised to one electron above $0.5 \mathrm{MeV}$, and we assume an upper cutoff energy of $100 \mathrm{MeV}$. The three separate curves signify the ICS spectrum as seen from three different viewing angles $\lambda$. In Fig. 3, we show the ICS spectra for an event viewed at the limb but now for different values of $\delta$.

Clearly visible $(2 \mathrm{eV})$ photons can easily be up-scattered to 10 s of keV, even though the actual fluxes and spectra depart from those expected on the basis of an isotropic photon distribution. 
Over most of the photon energy range the spectra are described by the expected (e.g. Blumenthal \& Gould 1970) ICS power-law $\sim \epsilon_{2}^{-(1+\delta) / 2}$, but falling off much more steeply as they approach an upper cutoff determined by the $100 \mathrm{MeV}$ electron upper cutoff, the viewing angle and the energy spectral index $\delta$. As expected on geometrical grounds, the most energetic photons come from limb events. In the photon energy range produced across the disc, ICS exhibits pronounced limb-brightening with flux variations of two orders of magnitude between identical events viewed at the limb and at disc centre. In Sect. 4 we see that observed coronal source photon fluxes imply plausible electron numbers.

A comparison of the ICS fluxes of Figs. 2 and 3 with the bremsstrahlung from the same electrons is not quite straightforward. For the usual monotonic declining energy distributions of electrons, the bremsstrahlung flux at some photon energy $\epsilon$ is dominated by electrons with energies just above $\epsilon$. ICS hard X-ray photons, however, are produced by electrons in the 10-100 s of MeV energy range. Any comparison of bremsstrahlung and ICS fluxes involves an assumption about the electron energy distribution over a very wide range. There is, for instance, evidence that electron distributions routinely harden between 10 s of $\mathrm{keV}$ and the $\mathrm{MeV}$ energy range (e.g. Silva et al. 2000). Including a bremsstrahlung spectrum for comparison in Fig. 2 could be quite misleading in consequence.

For illustration, we may nonetheless assume that a single power law distribution $\sim(\gamma-1)^{-\delta}$ in kinetic energy characterises the electron distribution all the way from $10 \mathrm{keV}$ to 100 s of MeV. Adopting $\delta=3$, for example, we find that the bremsstrahlung flux at $10 \mathrm{keV}$ will be comparable to the ICS flux for an ambient density of about $10^{10} \mathrm{~cm}^{-3}$. The harder ICS flux will dominate at photon energies above this value, until we approach the upper cutoff shown in Fig. 4. Thus ICS appears likely to dominate over bremsstrahlung for much of the time in the corona.

Still higher photon energies will result from primary photons of higher energy. For illustration we show in Fig. 4 the spectrum resulting from ICS of primary EUV photons of an energy of $200 \mathrm{eV}$, from a flare at disc centre. For easy comparison with the results for optical photons we have adopted the same photon density, $n_{\gamma}=10^{12} \mathrm{~cm}^{-3}$, although the true EUV density will be many orders of magnitude smaller - see below.

\subsection{ICS from relativistic positrons}

As noted in Sect. 1, positrons will be produced as secondaries from fast ion reactions. Electrons and positrons with the same energy distribution would of course produce identical ICS spectra, but the positron energy distribution from $\mathrm{p}-\mathrm{p}$ collisions and hence the ICS photon spectrum is quite different from the powerlaw electron case considered in Sect. 3.1. We calculate positron energy distributions as in Vilmer et al. (2003), which in turn closely follows Dermer (1986a,b), assuming they are produced via pion decay following reactions of fast protons with ambient $\mathrm{H}$ and $\mathrm{He}$ nuclei. The nuclear reactions producing the positrons occur mostly in the chromosphere and photosphere, but with a range of directions. At the energies considered here, any that mirror above the photosphere will suffer only an insignificant energy loss as they make their way into the corona (MacKinnon \& Brown 1990). For simplicity, we assume here that we may use the positron energy distribution from pion decay unmodified by any other processes. A more detailed treatment of transport will be carried out elsewhere. We see in Sect. 4 that only a few percent of the number of positrons produced in a large flare will give a detectable ICS source.

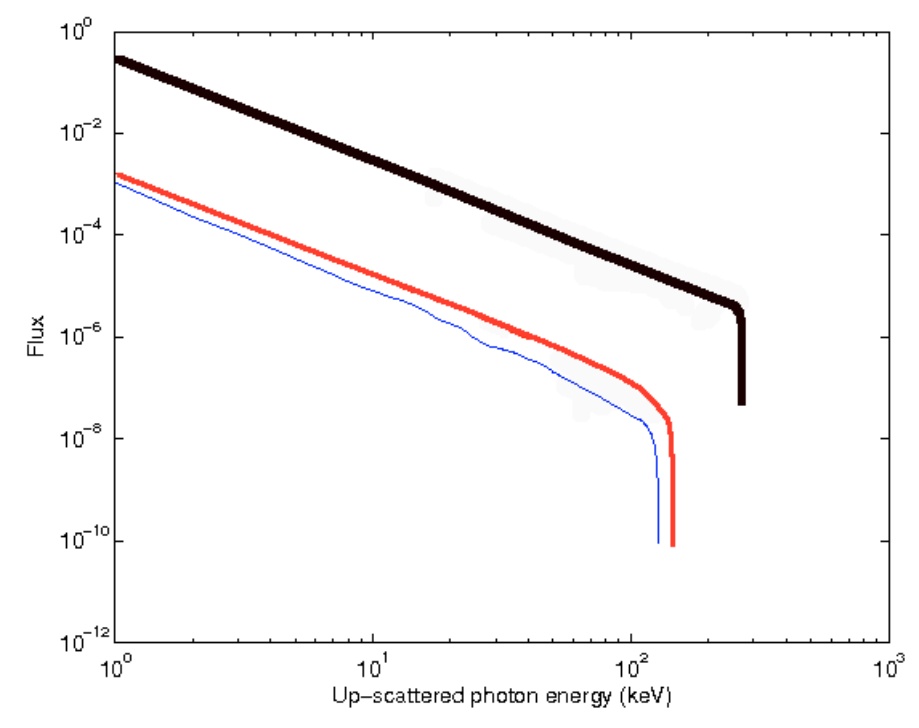

Fig. 4. Photon spectra at the Sun (photons per keV per second per source electron) from fast electrons with a power-law energy distribution $E^{-3}$, where the thin-blue curve is the flux from the disc centre $(\sin \lambda=0)$, medium-red for $\sin \lambda=0.5$ and thick-black for the solar limb $(\sin \lambda=$ 1). Fluxes are normalised to one electron above $0.5 \mathrm{MeV}$ and for an incident photon of energy $2 \mathrm{eV}$.

Positrons may also be produced in flares via beta decay of unstable nuclei produced in nuclear reactions of flare ions. As noted by Kozlovsky et al. (1987), positrons produced in this way generally have energies of $<1 \mathrm{MeV}$, too low to be of interest here.

ICS spectra from the resulting positrons are shown in Fig. 5, assuming a power-law proton energy distribution with $\delta=3$ extending to an upper cutoff energy of $3 \mathrm{GeV}$ and, again, $\epsilon_{1}=2 \mathrm{eV}$ and $n_{\gamma}=10^{12} \mathrm{~cm}^{-3}$. Secondary positron distributions have a maximum at about $300 \mathrm{MeV}$ and a form that is dominated by the nuclear physics of pion formation and decay until primary proton energies significantly exceed the threshold for pion production (Murphy et al. 1987). Thus the detailed photon spectra depend rather weakly on the proton power-law energy spectral index. However, certain features persist, i.e. the spectrum remains very hard, and the most energetic photons will once again come from limb events. The three separate curves are for three different values of the viewing angle $\lambda$. Also shown is the dashed-green curve in Fig. 5, which is the bremsstrahlung spectrum from the same positrons, assuming an ambient density of $10^{10} \mathrm{~cm}^{-3}$. We used the cross-section of Bethe and Heitler, without making non-relativistic or extreme relativistic approximations (Koch \& Motz 1959, formula 3BN) and the relativistic electron-electron cross-section of Haug (1998), noting that electron-electron and electron-positron cross-sections become identical for relativistic energies (Haug 1985). As mentioned above, the form of the positron distribution depends rather weakly on assumptions about the primary ion distribution, so this comparison can be made with much more certainty than for electrons. Even with this ambient density, fairly high for the corona, ICS dominates over the bremsstrahlung flux from the same positrons. Annihilation of positrons in flight yields a continuum photon flux that may be neglected compared to bremsstrahlung, for present purposes (Murphy et al. 1987).

In Fig. 6, we show the ICS spectra for a range of proton energy distribution $\delta$ values. Secondary positron typical energies naturally result in up-scattering to the $\mathrm{MeV}$ photon energy range. 


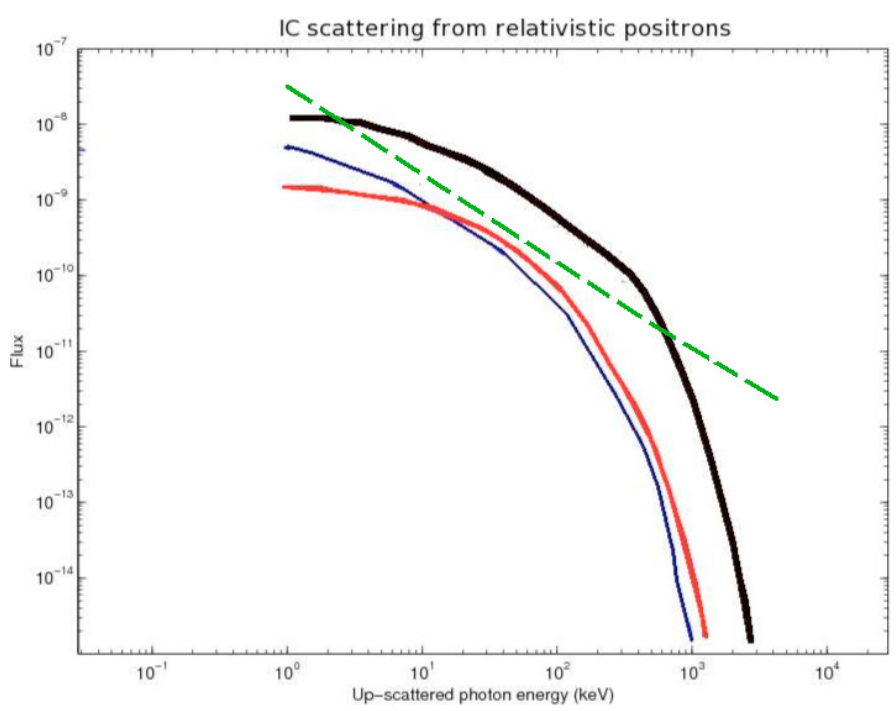

Fig. 5. Photon spectra at the Sun (photons per keV per second per proton) from relativistic positrons produced by protons with a power-law energy distribution $E^{-3}$, where the thin-blue curve is the flux from the disc centre $(\sin \lambda=0)$, medium-red for $\sin \lambda=0.5$ and thick-black for the solar limb flux $(\sin \lambda=1)$. Fluxes are normalised to one proton above $1 \mathrm{MeV}$ and for an incident photon energy of $2 \mathrm{eV}$. The dashed-green line represents the bremsstrahlung flux from the same positron distribution and has been included here to compare with the predicted ICS fluxes. Note that due to the extreme relativistic nature of the positrons, the bremsstrahlung flux is hard with an index of 1 . In other words, for such energetic positrons, their distribution has little effect on the spectral index of the bremsstrahlung radiation they produce.

As for the electron case, we would expect a more energetic ICS flux if we consider incident EUV photons, shown in Fig. 7 for $200 \mathrm{eV}$ incident photons. With the photon density held fixed, as for Fig. 5, the ICS flux can be as much as four orders of magnitude greater for $\epsilon_{1}=200 \mathrm{eV}$ than for $\epsilon_{1}=2 \mathrm{eV}$, at the same time extending to higher energies. So we would need an EUV photon density $\sim 10^{-4}$ times that of visible photons to produce an equally intense ICS flux. A rough estimate of EUV photon density in a large flare suggests this will be $\sim 10^{3} \mathrm{~cm}^{-3}$, however, so low that even the greater fluxes obtained with more energetic incident photons will not be observable.

\section{Comparison with observations}

As explained in Sect. 1, ICS could be dominant in producing HXRs in low-density regions of the solar atmosphere, which mainly implies the high corona. Consider the coronal $\mathrm{X} / \gamma$-ray source in the 2005 January 20 flare, described by Krucker et al. (2008b). Could it be due to ICS of photospheric photons?

Continuum $\gamma$-radiation in the $100 \mathrm{MeV}$ energy range was observed from this flare by the SONG instrument on CORONAS-F. There is evidence for a pion decay contribution to the observed spectrum (Kuznetsov et al. 2005), which would also indicate the presence of $\sim 100 \mathrm{MeV}$ positrons. The flare was located towards the limb $\left(\mathrm{N} 14^{\circ} \mathrm{W} 61^{\circ} ; \sin \lambda=0.88\right)$, maximising the likelihood of observable ICS photons. Moreover, the location of the coronal X-ray source is high enough that ICS could be the dominant source of HXRs, given sufficient energetic electrons. The coronal source has a very hard spectrum, of a photon spectral in$\operatorname{dex} \approx 1.5$, consistent with the spectra found in Sect. 3. A photon spectral index of 1.5 would imply a relativistic electron spectral index of about 2. Continuation of the photon spectrum to at least

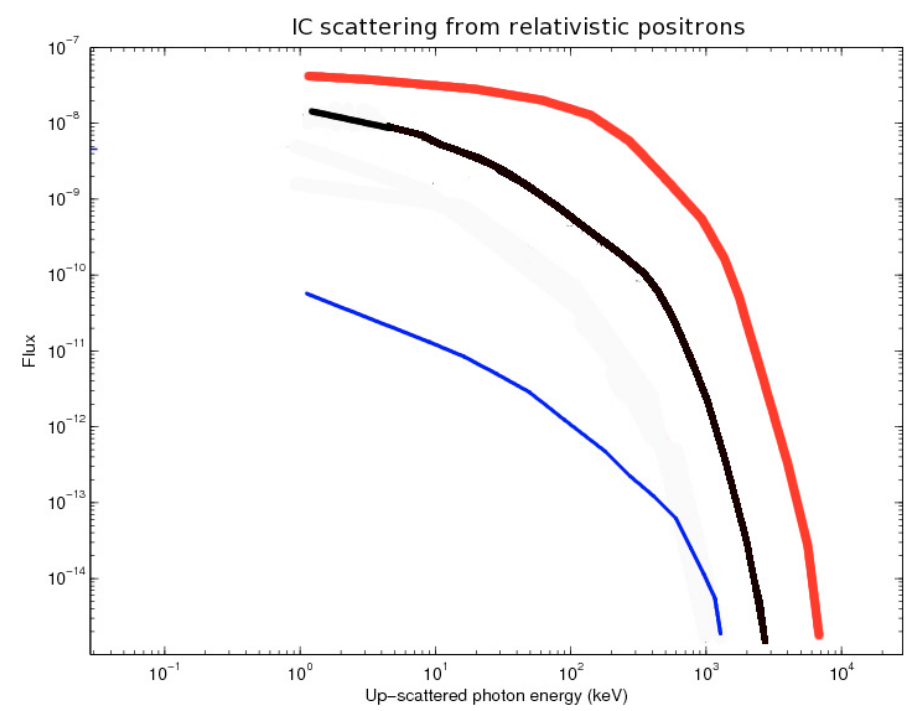

Fig. 6. Photon spectra at the Sun (photons per keV per second per proton) from limb relativistic positrons produced by protons with different power-law distributions: thin-blue is for $\delta=5$, medium-black for $\delta=3$ and thick-red for $\delta=2$ with an incident photon energy of $2 \mathrm{eV}$.

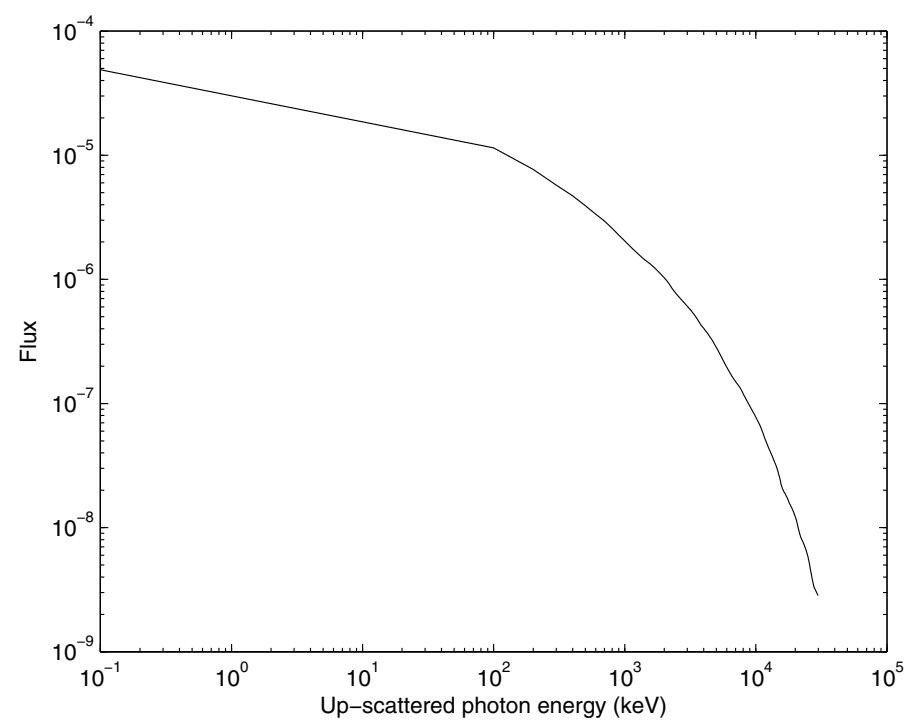

Fig. 7. Photon spectra at the Sun (photons per keV per second per proton) from relativistic positrons produced by protons with a power-law energy distribution $E^{-3}$ for an incident photon energy of $200 \mathrm{eV}$.

$700-800 \mathrm{keV}$ implies an electron distribution continuing in this power-law form to at least $120 \mathrm{MeV}$. To account for the observed coronal source fluxes shown in Fig. 3 of Krucker et al. (2008b), we would need $\sim 10^{31}$ electrons instantaneously present above $0.5 \mathrm{MeV}$. The $\sim 500 \mathrm{keV}$ source represented by the $50 \%$ contour of Krucker et al. (2008b), Fig. 2c, is about $40 \times 80 \mathrm{arcsec}$. Assuming a similar length scale along the line of sight we estimate its total volume as $5 \times 10^{28} \mathrm{~cm}^{3}$. Taking for illustration an ambient electron density $10^{8} \mathrm{~cm}^{-3}$, we see that the relativistic electrons necessary to account for this source via ICS would represent just $2 \times 10^{-6}$ of all particles in the volume. We also estimate that this is $\sim 10^{-3}$ or less of the electrons $>0.5 \mathrm{MeV}$ implied by a typical, large X-ray burst. An uncertain fraction of these would be trapped in the corona, and the electron distribution might not extend with the same energy dependence to 10 s of $\mathrm{MeV}$, but it appears quite plausible that enough electrons of the required energies are present in the flare. The minimum energy 
of $0.5 \mathrm{MeV}$ is of course quite arbitrary; only electrons in the $10 \mathrm{~s}$ of $\mathrm{MeV}$ range and above are demanded by an ICS interpretation of this coronal HXR source.

Close to the limb, the most favourable head-on collisions of electrons with primary photons may occur. The flux and spectrum are very close to those given traditionally for powerlaw electron distributions and isotropic primary photons (e.g. Blumenthal \& Gould 1970; Krucker et al. 2008a), with modifications resulting mostly from the presence of an upper electron cutoff energy. The number and energy distribution of electrons found above are close to those that would be found using the traditional results; but this would not be the case for an event further from the limb.

An interpretation in terms of positrons is also possible. The spectra shown in Fig. 5 would all give approximately the necessary hard spectrum in the several hundred keV energy range (although, as discussed above, none has precisely power-law form). For a power-law primary proton energy distribution with an energy spectral index $=2$, about $10^{32}$ protons would be needed above $1 \mathrm{MeV}$. Masson et al. (2009) found a proton flux of $2.3 \times 10^{31} \mathrm{~cm}^{-3}$ above $30 \mathrm{MeV}$ for this event and a proton spectral index $=3$, i.e. $2 \times 10^{34}$ protons above $1 \mathrm{MeV}$. Most secondary positrons presumably stop at great depths in the atmosphere, but we would need only a few percent of them to find their way into the corona in order to account for the coronal HXR source via ICS.

\section{Conclusions and discussion}

ICS needs extreme source parameters if it is to account on its own for the bulk of flare hard X-rays (Korchak 1971; McClements \& Brown 1986), particularly when 'footpoint' source morphology points to an origin in the dense chromosphere. Our work does not revise this view, just points out that ICS might be important for understanding sources in the tenuous corona. We have seen that very modest numbers of electrons or positrons at relativistic energies could account for already observed coronal HXR sources, even in regions so tenuous that a conventional bremsstrahlung interpretation would become problematic. Electrons would need to be accelerated into the $100 \mathrm{MeV}$ energy range; positrons are automatically produced with the necessary energies as long as there are $\sim \mathrm{GeV}$ protons to produce them in the first place. The electron distribution needed to account for HXR bursts, extended into the $100 \mathrm{MeV}$ energy range, would include enough relativistic electrons that only a small fraction of them would need to be found in the corona to account for at least one observed coronal HXR source. Moreover, electrons might be accelerated to relativistic energies via a process distinct from the main flare energy release, as appears to occur in the Earth's magnetosphere (Baker et al. 2001).

How might we distinguish these sources from conventional bremsstrahlung HXRs? First of all, they may be expected from locations where the ambient density seems too low for a conventional bremsstrahlung interpretation. As we have seen, coronal ICS sources should be brightest near the solar limb. If many sources like those described in Krucker et al. (2008b) can be detected, an ICS interpretation would imply a strong centre-to-limb variation. Simultaneous observations from two widely separated spacercraft (e.g. Krucker et al. 2008b) would reveal quite different fluxes and spectra. The spectra will always be very hard, possibly also extending to soft X-ray and EUV ranges in a continuous way difficult to account for by other means. Observations of co-spatial radio radiation would have very different spectral properties in the bremsstrahlung and ICS cases.
Do we need to contain electrons in the corona to produce such sources? The calculations above assume that an isotropic population of electrons is instantaneously present in the source region. Radio observations show coronal containment of highenergy (gyrosynchrotron emitting) electrons (Kundu et al. 2001; Melnikov et al. 2002). The overwhelming contribution to observed ICS, however, comes from electrons moving instantaneously towards the observer. Electrons could pass freely through the corona, following the field lines and emitting observable ICS HXRs as they pass through the line of sight towards the observer. They would not need to be contained in the corona, and an isolated coronal source might be more naturally explained in this way, as a consequence of relativistic beaming and source magnetic geometry. Instantaneous numbers of electrons needed would be comparable to the numbers found above. A more detailed treatment of electron and positron transport, not given here, would be needed to assess this possibility properly.

Our assumed isotropic electron distribution raises similar questions. We appealed to electron and positron scattering by MHD turbulence to justify this assumption (e.g. Miller \& Ramaty 1989). It still seems unclear if the coronal electron trapping revealed in radio is due to turbulence, magnetic field convergence and/or other physical factors. Electrons may be coronally contained but anisotropic. The consequences of anisotropy are more easily addressed for our highly relativistic electrons than e.g. the study of gyrosynchrotron radiation carried out by Fleishman \& Melnikov (2003). The cone of emission about the electron instantaneous direction of motion has width $\gamma^{-1}$, so the electron distribution function and the loop geometry (e.g. orientation north-south; any tilt to the vertical, etc. - cf. MacKinnon \& Brown 1990) would have to conspire to ensure that some electrons travel more or less in the line of sight. Deduced numbers of electrons would be of the same order of magnitude as those found assuming isotropy, but the range of viewing angles giving rise to an observable source would be narrower.

ICS coronal X-ray sources may already have been observed. Already well-studied sources, e.g. in the Masuda flare (Masuda et al. 1994) or some of those described by Tomczak (2009), might be reinterpreted in this way. In these smaller events, including the $\mathrm{M}$ class Masuda flare, there are no $\gamma$-ray measurements to give any independent constraint on high-energy electrons or positrons, however. In small flares, bright coronal HXR sources in implausibly tenuous regions would indicate the presence of relativistic electrons or positrons.

Might ICS yield observable contributions in other wavelength ranges? Flare positrons, for example, would scatter $\mathrm{cm}$ wavelength photons into the optical or near UV ranges. In the corona the primary photon number density would be extremely low, making an observable flux highly unlikely, unless the relativistic electrons or positrons lay within an optically thick microwave source. Such a situation would need a much more detailed evaluation of the primary radiation field than we have carried out here, along the lines of McClements \& Brown (1986). Other possibilities, like an ICS contribution to the flare UV continuum, appear potentially interesting but would take place in the deeper atmosphere and would similarly require a different treatment of the primary radiation field.

If definitively recognised in flares, ICS coronal HXR sources would open a new window on acceleration and transport of electrons and ions in the $0.1-1 \mathrm{GeV}$ energy range.

Acknowledgements. The initial impetus for this work came from the I.S.S.I. International Team on "Coronal Hard X-ray Sources in Solar Flare". A.L.M. thanks ISSI for hosting the Team; and S. Krucker, H. Hudson and the other 
A. L. MacKinnon and P. C. V. Mallik: Inverse Compton flare X-rays

participants for discussion. Conversations with T. Porter were also useful. P.C.V.M. is supported by a UK STFC Dorothy Hodgkin's Scholarship. Solar physics research in Glasgow is supported by an STFC Rolling Grant and by the European Union via the SOLAIRE Network (MTRN-CT-2006-035484).

\section{References}

Akimov, V. V., Leikov, N. G., Kurt, V. G., \& Chertok, I. M. 1994, in High-Energy Solar Phenomena - a New Era of Spacecraft Measurements, ed. J. Ryan, \& W. T. Vestrand, AIP Conf. Ser., 294, 130

Baker, D. N., Kanekal, S. G., Blake, J. B., \& Pulkkinen, T. I. 2001, J. Geophys. Res., 106, 19169

Blumenthal, G. R., \& Gould, R. J. 1970, Rev. Mod. Phys., 42, 237

Brown, J. C., \& Mallik, P. C. V. 2008, A\&A, 481, 507

Brown, J. C., \& Mallik, P. C. V. 2009, ApJ, 697, L6

Dermer, C. D. 1986a, ApJ, 307, 47

Dermer, C. D. 1986b, A\&A, 157, 223

Fleishman, G. D., \& Melnikov, V. F. 2003, ApJ, 587, 823

Forrest, D. J., \& Chupp, E. L. 1983, Nature, 305, 291

Haug, E. 1985, Phys. Rev. D, 31, 2120

Haug, E. 1998, Sol. Phys., 178, 341

Hudson, H. S., Kosugi, T., Nitta, N. V., \& Shimojo, M. 2001, ApJ, 561, L211

Jauch, J. M., \& Rohrlich, F. 1976, The theory of photons and electrons, The relativistic quantum field theory of charged particles with spin one-half, 2nd edn. (New York: Springer)

Jones, F. C. 1968, Phys. Rev., 167, 1159

Kanbach, G., Bertsch, D. L., Fichtel, C. E., et al. 1993, A\&AS, 97, 349

Koch, H. W., \& Motz, J. W. 1959, Rev. Mod. Phys., 31, 920
Korchak, A. A. 1967, SvA, 11, 258

Korchak, A. A. 1971, Sol. Phys., 18, 284

Kozlovsky, B., Lingenfelter, R. E., \& Ramaty, R. 1987, ApJ, 316, 801

Krucker, S., Battaglia, M., Cargill, P. J., et al. 2008a, A\&AR, 16, 155

Krucker, S., Hurford, G. J., MacKinnon, A. L., Shih, A. Y., \& Lin, R. P. 2008b, ApJ, 678, L63

Kundu, M. R., Nindos, A., White, S. M., \& Grechnev, V. V. 2001, ApJ, 557, 880 Kuznetsov, S. N., Kurt, V. G., Yushkov, B. Y., et al. 2005, in Int. Cosmic Ray Conf., 1, 49

MacKinnon, A. L., \& Brown, J. C. 1990, A\&A, 232, 544

Mandzhavidze, N., \& Ramaty, R. 1992, ApJ, 389, 739

Masson, S., Klein, K.-L., Bütikofer, R., et al. 2009, Sol. Phys., 257, 305

Masuda, S., Kosugi, T., Hara, H., Tsuneta, S., \& Ogawara, Y. 1994, Nature, 371, 495

McClements, K. G., \& Brown, J. C. 1986, A\&A, 165, 235

Melnikov, V. F., Shibasaki, K., \& Reznikova, V. E. 2002, ApJ, 580, L185

Miller, J. A., \& Ramaty, R. 1989, ApJ, 344, 973

Moskalenko, I. V., \& Strong, A. W. 2000, ApJ, 528, 357

Murphy, R. J., Dermer, C. D., \& Ramaty, R. 1987, ApJS, 63, 721

Orlando, E., \& Strong, A. W. 2008, A\&A, 480, 847

Rybicki, G. B., \& Lightman, A. P. 1986, Radiative Processes in Astrophysics (Wiley-VCH)

Silva, A. V. R., Wang, H., \& Gary, D. E. 2000, ApJ, 545, 1116

Silva, A. V. R., Share, G. H., Murphy, R. J., et al. 2007, Sol. Phys., 245, 311

Sunyaev, R. A., \& Zeldovich, Y. B. 1970, Ap\&SS, 7, 3

Talon, R., Trottet, G., Vilmer, N., et al. 1993, Sol. Phys., 147, 137

Tomczak, M. 2009, A\&A, 502, 665

Vilmer, N., MacKinnon, A. L., Trottet, G., \& Barat, C. 2003, A\&A, 412, 865

Weaver, T. A. 1976, Phys. Rev. A, 13, 1563 